\title{
On asymmetric colourings of claw-free graphs*
}

\author{
Wilfried Imrich \\ Montanuniversität Leoben, A-8700 Leoben, Austria \\ imrich@unileoben.ac.at \\ Rafał Kalinowski Monika Pilśniak Mariusz Woźniak \\ AGH University of Science and Technology, 30-059 Krakow, Poland \\ \{kalinows, pilsniak, mwozniak\}@agh.edu.pl
}

Submitted: Jul 19, 2019; Accepted: Jul 4, 2021; Published: Jul 16, 2021

(C) The authors. Released under the CC BY-ND license (International 4.0).

\begin{abstract}
A vertex colouring of a graph is asymmetric if it is preserved only by the identity automorphism. The minimum number of colours needed for an asymmetric colouring of a graph $G$ is called the asymmetric colouring number or distinguishing number $D(G)$ of $G$. It is well known that $D(G)$ is closely related to the least number of vertices moved by any non-identity automorphism, the so-called motion $m(G)$ of $G$. Large motion is usually correlated with small $D(G)$. Recently, Babai posed the question whether there exists a function $f(d)$ such that every connected, countable graph $G$ with maximum degree $\Delta(G) \leqslant d$ and motion $m(G)>f(d)$ has an asymmetric 2-colouring, with at most finitely many exceptions for every degree.

We prove the following result: if $G$ is a connected, countable graph of maximum degree at most 4 , without an induced claw $K_{1,3}$, then $D(G)=2$ whenever $m(G)>2$, with three exceptional small graphs. This answers the question of Babai for $d=4$ in the class of claw-free graphs.
\end{abstract}

Mathematics Subject Classifications: 05E15, 05C15

\section{Introduction}

We consider countable graphs, that is, graphs with finite or denumerable vertex sets, and use standard graph theoretic notation. A colouring, not necessarily proper, of a graph $G$ is called asymmetric or distinguishing if it is preserved only by the identity automorphism of $G$. The asymmetric colouring number or distinguishing number $D(G)$ of a graph $G$ is the least number of colours in an asymmetric vertex colouring, and the distinguishing

${ }^{*}$ The research was partially supported by OEAD grant no. PL 08/2017. 
index $D^{\prime}(G)$ is the least number of colours in an asymmetric edge colouring of $G$. The vertex invariant $D(G)$ was defined in [1], and the edge invariant $D^{\prime}(G)$ in [11].

The motion $m(\varphi)$ of an automorphism $\varphi$ of a graph $G$ is the number of vertices that $\varphi$ moves, that is, the number of vertices $v$ for which $\varphi(v) \neq v(\operatorname{if} \operatorname{Aut}(G)=\{\operatorname{id}\}$, then we set $m(G)=0)$. The motion $m(G)$ of a graph $G$ is the minimum motion of a nonidentity automorphism of $G$. A relationship between the distinguishing number and the motion of a graph was observed 1998 by Russell and Sundaram in [19], who proved that $D(G) \leqslant d$ whenever $m(G) \geqslant 2 \log _{d}|\operatorname{Aut}(G)|$, but we wish to observe that, essentially, this was already shown 1986 in a different context by Cameron in [5].

Perhaps, the most intriguing open problem in this area is the Infinite Motion Conjecture of Tucker [20], who conjectured that $D(G) \leqslant 2$ for every connected, locally finite graph $G$ if every nontrivial automorphism of $G$ moves infinitely many vertices. The conjecture is open, though it was proved to be correct for several classes of graphs. For instance, Lehner, Pilśniak and Stawiski [14] recently confirmed this conjecture for graphs $G$ of maximum degree $\Delta(G) \leqslant 5$ (the case $\Delta(G)=3$ was independently proved in [7]).

In 2017, Lehner [13] settled affirmatively the Infinite Edge-Motion Conjecture of Broere and Pilśniak [4] that $D^{\prime}(G) \leqslant 2$ for every countable, connected graph $G$ if every nonidentity automorphism of $G$ moves infinitely many edges. This implies the validity of the Infinite Motion Conjecture of Tucker for line graphs. A classical theorem of Beineke [3] characterizes line graphs by nine excluded induced subgraphs. One of them is the claw $K_{1,3}$. Hence, a natural extension of the result of Lehner would be a confirmation of the Infinite Motion Conjecture for claw-free graphs.

Another motivation for our research was the following question posed by Babai in 2018 .

Question 1. (Babai [2]) Does there exists a function $f(d)$ such that $D(G)=2$ for every connected, countable graph $G$ with maximum degree $\Delta(G) \leqslant d$ and motion $m(G)>f(d)$.

This question for $d=3$ was fully answered in a recent paper of Hüning, Imrich, Kloas, Schreiber and Tucker [7], who gave a complete classification of all countable connected graphs $G$ of maximum degree $\Delta(G)=3$ and distinguishing number $D(G) \geqslant 3$. As a consequence, they observed that the only subcubic graphs $G$ with $D(G)>2$ and $m(G)>2$ are the cube $K_{2} \square K_{2} \square K_{2}$ and the Petersen graph, both containing an induced claw. They also posed the question whether all but finitely many graphs $G$ with the property that $m(G)>\Delta(G) \geqslant 4$ have distinguishing number 2 .

For trees $f(d)=2\left\lceil\log _{2} d\right\rceil$, see [9].

Let us also mention that Lehner and Verret [16] recently determined all finite, 4-regular, connected, vertex-transitive graphs $G$ with $D(G)>2$. These are $K_{5}, K_{3} \square K_{3}$, $K_{4} \square K_{2}, K_{5} \times K_{2}$ and an infinite family of lexicographic products $C_{n}\left[2 K_{1}\right], n \geqslant 3$, called wreath graphs.

The above-mentioned results were the motivation for the following theorem, which is the main result of our paper.

Theorem 2. Let $G$ be a countable, connected, claw-free graph of maximum degree $\Delta \leqslant 4$ and motion $m(G)$, where $4 \leqslant m(G)$. Then $D(G)=2$, unless $G \in\left\{C_{5}, K_{3} \square K_{3}, K_{4} \square K_{2}\right\}$. 
Note that the condition $m(G) \geqslant 4$ is equivalent to $m(G)>2$. In other words, $m(G) \neq 3$ for any graph $G$. Indeed, suppose $m(\varphi)=3$ for some $\varphi \in \operatorname{Aut}(G)$, which means that $\varphi$ moves three vertices. By considering $\varphi^{2}$, it is easily seen that the closed neighbourhood of each of these three vertices is the same. Thus, a transposition of any two of them is an automorphism of $G$. Hence, $m(G)=2$.

Our result is sharp since there are infinitely many claw-free graphs $G$ with $D(G)=3$ while $\Delta(G)=4$ and $m(G)=2$. For instance, consider a graph $G$ obtained by attaching a path of any length, also infinite, to one vertex of $K_{4}$.

We conjecture that our result can be generalized to higher maximum degrees.

Conjecture 3. Let $G$ be a countable, connected, claw-free graph with finite maximum degree $\Delta(G) \leqslant m(G)$. Then $D(G)=2$, unless $G$ is one of finitely many exceptional graphs.

\section{Auxiliary results}

In this section we present several known results which we use in the proof of Theorem 2 .

We begin with the following general upper bound for the distinguishing number, proved by Collins and Trenk [6], and independently by Klavžar, Wong and Zhu [12].

Theorem 4. ([6], [12]) If $G$ is a finite, connected graph with maximum degree $\Delta(G)$, then $D(G) \leqslant \Delta(G)+1$. Moreover, the equality is achieved if and only if $G$ is a $K_{n}, K_{p, p}$ or $C_{5}$.

For infinite graphs, Lehner, Pilśniak and Stawiski [14] recently obtained a better, sharp upper bound, thus improving a previous bound proved by Imrich, Klavžar and Trofimov [8].

Theorem 5. ([14]) If $G$ is an infinite, connected graph with finite maximum degree $\Delta(G) \geqslant 3$, then $D(G) \leqslant \Delta(G)-1$.

A general upper bound for the distinguishing index was established by Kalinowski and Pilśniak [11].

Theorem 6. ([11]) If $G$ is a finite, connected graph, then $D^{\prime}(G) \leqslant \Delta(G)$ unless $G$ is a cycle of length at most five.

Next, Pilśniak [17] characterized the graphs $G$ satisfying the equality $D^{\prime}(G)=\Delta(G)$. A tree $T$ is called symmetric if it has a central vertex $v_{0}$, all leaves have the same distance from $v_{0}$, and all vertices that are not leaves have the same degree. Substituting a central vertex $v_{0}$ by a central edge $e_{0}$ one obtains a bisymmetric tree.

Theorem 7. ([17]) For finite, connected graphs $G$, the equality $D^{\prime}(G)=\Delta(G)$ holds only for symmetric and bisymmetric trees, cycles of length at least six, for $K_{4}$ and $K_{3,3}$. 
For infinite graphs, Pilśniak and Stawiski [18] showed that $D^{\prime}(G) \leqslant \Delta(G)-1$ whenever $G$ is connected and $\Delta(G) \geqslant 3$.

It is worth mentioning that Lehner and Smith in [15] discussed recently the relation between $D(G)$ and $D^{\prime}(G)$.

Analogously to the vertex case, the edge motion of a graph $G$, denoted by $m^{\prime}(G)$, is defined as the least number of edges moved by any non-identity automorphism.

The following generalization of Whitney's theorem proved by Jung [10] exibits the equivalence between distinguishing edge-colourings of a graph $G$ and distinguishing vertexcolourings of its line graph $L(G)$.

Theorem 8. ([10]) If $G$ is a connected graph of order greater than four, then the automorphism groups $\operatorname{Aut}(G)$ and $\operatorname{Aut}(L(G))$ are isomorphic by means of the natural map from $\operatorname{Aut}(G)$ onto $\operatorname{Aut}(L(G))$.

This theorem is also valid for infinite graphs, and it clearly implies the subsequent fact.

Corollary 9. If $G$ is a connected graph of order greater than four, then $D^{\prime}(G)=D(L(G))$ and $m^{\prime}(G)=m(L(G))$.

The following characterization of line graphs is due to Beineke [3]. Although it was stated for finite graphs, it is obviously true for infinite graphs, as well.

Theorem 10. ([3]) Let $G$ be a graph. There exists a graph $H$ such that $G$ is a line graph of $H$ if and only if $G$ contains no induced subgraph isomorphic to a claw nor to any of the eight graphs $B_{i}, i=1, \ldots, 8$, presented in Figure 2 and Figure 3 in Section 4.

\section{Distinguishing index of selected graphs of bounded degrees}

In this section we prove a lemma that will be useful in the proof of our main result for line graphs.

Lemma 11. Let $H$ be a connected, countable graph of order at least five such that $\Delta(L(H))=4$ and $m^{\prime}(H)>2$. Then $D^{\prime}(H)=2$, unless $H$ is $K_{3,3}$ or $K_{4,2}$.

Proof. We colour the edges of $H$ with two colours, blue and green. The assumption $\Delta(L(H))=4$ implies that $d_{H}(x)+d_{H}(y) \leqslant 6$ for every edge $x y$ in $H$. It follows that $\Delta(H) \leqslant 4$ unless $H=K_{1,5}$, but $m^{\prime}\left(K_{1,5}\right)=2$. If $\Delta(H) \leqslant 3$, then we infer from Theorem 6 and Theorem 7 that $D^{\prime}(H) \leqslant 2$ unless $H=K_{3,3}$. Indeed, the edge motion of any symmetric or bisymmetric tree $T$ equals two whenever $\Delta(T) \geqslant 3$.

Let then $\Delta(H)=4$. If $x$ is a vertex of degree 4 in $H$, then all its neighbours are of degree one or two. Moreover, the four neighbours are independent and at most one of them is a pendant vertex, because $m^{\prime}(H)>2$. Therefore, either there are four paths of length 2 (called arms) going out from $x$, or only three such paths and one pendant edge. In the first case we call such a vertex four-armed. Note that end-vertices of the 
arms may coincide. A four-armed vertex is fully coloured if its arms are coloured with all four possible sequences of colours.

The idea of the proof is to recursively construct an asymmetric edge colouring $c$ of the graph $H$. We start by choosing a vertex $v_{0}$ of degree 4 and colour its arms and a possible incident pendant edge in such a way that $v_{0}$ will be the only vertex of $H$ with such a colouring. Therefore, $v_{0}$ will be fixed by every automorphism of $H$ preserving the colouring $c$ (later on, we write fixed by $c$, for short). Thus, we consider an initial subgraph $F_{0}$ induced by all vertices of distance at most 2 from $v_{0}$. We colour the edges of $F_{0}$ in such a way that each vertex of $F_{0}$ will be fixed by $c$. Then, we consider a sequence of induced subgraphs $F_{1}, F_{2}, \ldots$ of $H$ such that $F_{i-1}$ is a proper subgraph of $F_{i}$ for $i=1,2, \ldots$ Namely, $F_{i}$ is a subgraph induced by the vertices of $F_{i-1}$, their neighbours and vertices of arms incident to vertices of degree 4 of $F_{i-1}$. It is easily seen that if we have defined an edge colouring of $F_{i-1}$ fixing each vertex of $F_{i-1}$, then $F_{i}$ must be fixed setwise. We extend the colouring to a suitable colouring of $F_{i}$ leaving the colours of edges of $F_{i-1}$ unchanged, and such that the subgraph $F_{i}$ is fixed pointwise.

Case 1. Let us first assume that $H$ contains a vertex $v_{0}$ of degree 4 that is not four-armed. We colour its three arms with distinct sequences of colours, and its incident pendant edge we colour with blue. In our colouring $c$ of edges of the whole graph $H$, we ensure that $v_{0}$ is a unique vertex of degree 4 with a blue incident pendant edge. Thus, $v_{0}$ will be fixed by $c$. Consequently, all the vertices of the subgraph $F_{0}$ induced by $v_{0}$, its neighbours and end-vertices of its arms, are also fixed by $c$. We colour the edges joining them arbitrarily.

We recursively define an edge colouring $c$ of $H$ as follows. Let $i \geqslant 1$, and assume that we have already coloured edges of a connected induced subgraph $F_{i-1}$ of $H$ such that $v_{0}$ is the only vertex of degree 4 in $F_{i-1}$ with a blue incident pendant edge, and the only automorphism of $F_{i-1}$ preserving this colouring is the identity. Consequently, each vertex of $F_{i-1}$ will be fixed by every automorphism of $H$ preserving any extension $c$ of this partial colouring, provided $v_{0}$ remains a unique vertex of degree 4 in $H$ with a blue incident pendant edge.

To extend this colouring we consecutively consider all vertices $v \in V\left(F_{i-1}\right)$ having neighbours outside $F_{i-1}$. At every stage of our colouring procedure, each vertex with a coloured incident edge becomes fixed by $c$.

First we consider vertices $v$ with $d(v)=2$, i.e. with at most one neighbour $u$ outside $F_{i-1}$. Then $u$ is also fixed by $c$ since it is a unique neighbour outside $F_{i-1}$ of a fixed vertex $v$. We can colour the edge $v u$ arbitrarily. If $d(v)=3$ and $v$ has two incident edges outside $F_{i-1}$, then we colour them with two different colours. If $v$ has only one neighbour $u$ outside $F_{i-1}$, then $u$ is fixed, and we colour $v u$ arbitrarily. In both cases, the neighbours of $v$ are fixed. At last, we consider vertices $v$ with $d(v)=4$. If $v$ has a pendant edge $v u$, then $u \notin V\left(F_{i-1}\right)$, and we colour $u v$ with green. If $v$ has arms outside $F_{i-1}$, then we colour them with distinct pairs of colours.

In all the above situations, our colouring fixes all considered (not yet fixed) vertices, 
i.e. vertices of $F_{i}-V\left(F_{i-1}\right)$ with at least one coloured incident edge. At the end of this stage, we colour each yet uncoloured edge between these vertices arbitrarily.

Thus we have coloured the edges of a larger subgraph $F_{i}$ which in the next step plays the role of $F_{i-1}$. After a countable number of steps, we get a desired asymmetric edge colouring $c$ of the whole graph $H$ (where $v_{0}$ is the only vertex of degree 4 with a blue pendant edge).

Case 2. Assume now that all vertices of degree 4 in $H$ are four-armed. We choose any such vertex $v_{0}$ of degree 4 and fully colour its arms. In our colouring $c$ of all edges of $H$, we ensure that $v_{0}$ is the only fully coloured vertex of degree 4 . This guarantees that $v_{0}$ will be fixed by the colouring $c$. Note that then also the vertices lying on the arms of $v_{0}$ will be fixed by $c$. Now we have to colour the remaining edges of the graph $F_{0}$, induced by $v_{0}$ and the vertices on its arms. All vertices of the graph $F_{0}$ are fixed, so the colour of these edges does not matter for asymmetry, but it may happen that some edges are incident with another vertex of degree 4, and we must ensure that this vertex is not fully coloured. We shall call each such edge a finishing edge. Figure 1 shows all three possible such situations with suitable colourings. If four arms of $v_{0}$ meet at one vertex, then $F_{0}=H=K_{4,2}$, an excluded graph with $D^{\prime}\left(K_{4,2}\right)=3$. In all other cases, $v_{0}$ is the only fully coloured vertex of degree 4 in $F_{0}$. Hence, since $v_{0}$ is fixed by $c$, the subgraph $F_{0}$ is also fixed pointwise.
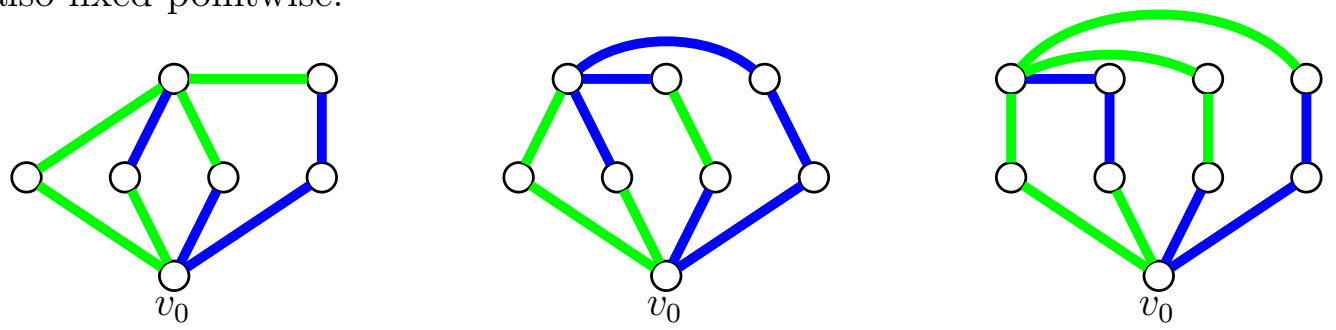

Figure 1: The vertex $v_{0}$ is the only fully coloured vertex of degree 4 in $F_{0}$.

We recursively colour all edges of $H$ similarly as in Case 1. However, now our task is a bit more difficult because we have to be careful not to create any new fully coloured vertex of degree 4. If a colouring of an arm may cause that some vertex of degree 4 becomes fully coloured, we call it a finishing arm.

Again, assume that we have already coloured edges of a connected induced subgraph $F_{i-1}$ such that $v_{0}$ is the only fully coloured vertex in $F_{i-1}$, and the only automorphism of $F_{i-1}$ preserving this colouring is the identity. Consequently, each vertex of $F_{i-1}$ will be fixed by every automorphism of $H$ preserving any extension $c$ of this partial colouring, provided $v_{0}$ remains a unique fully coloured vertex in $H$.

Suppose that a vertex $v$ of degree 2 has a neighbour $u$ outside $F_{i-1}$. If the edge $u v$ is finishing for $u$, then we colour $u v$ such that $u$ is not a fully coloured vertex. Otherwise, we can colour $u v$ arbitrarily, and $u$ is fixed by $c$. If $d(v)=3$, then none of its incident edges can be finishing, and we proceed in the same way as in Case 1.

Finally, we consider vertices $v$ with $d(v)=4$. If $v$ has only one arm outside $F_{i-1}$, say $v x u$, the three other arms incident to $v$ are already coloured. If this arm is not finishing 
for $u$, then we colour it using a sequence of colours already used for another arm of $v$. If this arm is finishing, then we have to use a sequence of colours which is already used for an arm of $v$ as well as for an arm of $u$. This is always possible since at most two colourings of the arm vxu may create a fully coloured vertex, $v$ or $u$, and there are four possible colourings of this arm.

Now, let $v$ have two arms outside $F_{i-1}$, say $v x_{1} u_{1}$ and $v x_{2} u_{2}$. Let us consider first the case when both arms are finishing. If $u_{1} \neq u_{2}$, we first colour the arm $v x_{1} u_{1}$ in such a way that the vertex $u_{1}$ is not fully coloured, which is again an easy task. Thus, only one arm $v x_{2} u_{2}$ is left, and we proceed with this arm as in the previous paragraph. If $u_{1}=u_{2}$, then we colour the arm $v x_{1} u_{1}$ with a colouring used for another already coloured arm incident to $v$. Hence, $v$ will not be fully coloured. Next, we colour the arm $v x_{2} u_{1}$ with a colouring of an already coloured arm incident to $u$ which is different from that of $v x_{1} u_{1}$. Thus, $u$ is also not fully coloured.

If at least one these two arms, say $v x u_{2}$, is not finishing, then we first colour the arm $v x u_{1}$ as above, such that $u_{1}$ is not fully coloured. Next, we colour the arm $v x u_{2}$ with a colouring already used for another arm incident to $v$. Hence, $v$ is not fully coloured. If none of these two arms is finishing we colour them with two distinct colourings, using at least one colouring already used.

Finally, consider the case when $v$ has three arms outside $F_{i-1}$, say $v x_{1} u_{1}, v x_{2} u_{2}$ and $v x_{3} u_{3}$, and let us suppose that all arms are finishing. If $u_{1} \neq u_{2}$ and $u_{1} \neq u_{3}$, we first colour the arm $v x_{1} u_{1}$ in such a way that the vertex $u_{1}$ is not fully coloured, i.e. with an already used colouring of an arm at $u_{1}$. Thus, two arms incident to $v$ are uncoloured, and we proceed with them as in the previous paragraph. If $u_{1}=u_{2}=u_{3}$ we need to ensure that the colouring of these three arms is different and neither $v$ nor $u$ are fully coloured. One can easily check that it is always possible.

To complete the recursive step, we have to colour every uncoloured edge $u w$ between two vertices $u, w$, each with at least one incident newly coloured edge. If $u v$ is a finishing edge for one of these vertices, say $u$, then we colour it in such a way that $u$ is not fully coloured, i.e. it has at least two arms coloured identically. Otherwise, $u$ and $v$ are already fixed by $c$, and we can colour them arbitrarily.

This completes the recursive step. Finally, we get a desired asymmetric 2-colouring of edges of the whole graph $H$ because $v_{0}$ is the only fully coloured vertex in the colouring $c$ of $H$. Observe that this recursive procedure clearly also works for infinite graphs because $H=\bigcup_{i=0}^{\infty} F_{i}$, since $H$ is connected.

\section{Proof of the Main Theorem}

In this section we complete the proof of Theorem 2 .

Proof of Theorem 2. Let $G$ be a countable, connected claw-free graph with maximum degree $\Delta(G) \leqslant 4$ and motion $m(G) \geqslant 4$. If $\Delta(G)=2$, then, by Theorem $4, D(G) \leqslant 2$, unless $G$ is a cycle $C_{5}$, because $m\left(K_{3}\right)=m\left(K_{2,2}\right)=2$. As mentioned in the Introduction, 
it was proved in [7] that the only graphs $G$ with $\Delta(G)=3, m(G)>2$ and $D(G)>2$, are the cube and the Petersen graph. Both of these exceptional graphs contain a claw.

Let $\Delta(G)=4$. Suppose first that $G$ is the line graph of a graph $H$. If $|H| \leqslant 4$, then $H \in\left\{K_{4}, K_{4}-e\right\}$, because $\Delta(L(H))=4$. However, both graphs $L\left(K_{4}\right)$ and $L\left(K_{4}-e\right)$ have motion 2. If $|H| \geqslant 5$, then Lemma 11 implies that $D(G)=2$, unless $G=L\left(K_{3,3}\right)=$ $K_{3} \square K_{3}$ or $G=L\left(K_{4,2}\right)=K_{4} \square K_{2}$.

To complete the proof, we have to show that $D(G)=2$ for every countable claw-free graph $G$ such that $\Delta(G)=4, m(G) \geqslant 4$, and $G$ is not the line graph of any graph.
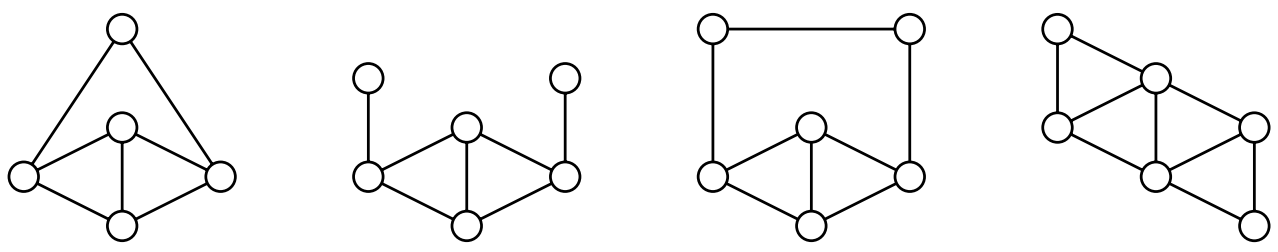

Figure 2: Beineke graphs $B_{1}, B_{2}, B_{3}, B_{4}$.
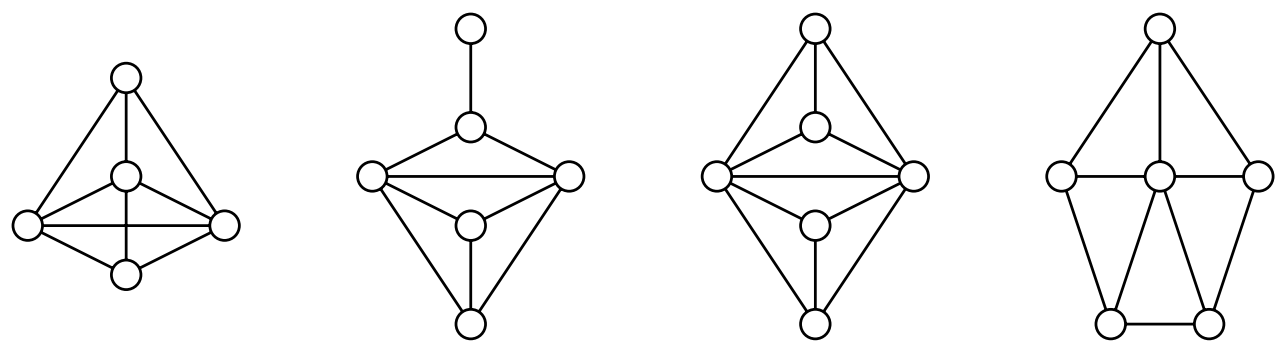

Figure 3: Beineke graphs $B_{5}, B_{6}, B_{7}, B_{8}$.

As $G$ is claw-free, it follows from Beineke's Theorem 10 that $G$ contains at least one of the eight graphs $B_{i}, i=1, \ldots, 8$, shown in Figure 2 and Figure 3, as induced subgraphs. The graphs $B_{7}$ and $B_{8}$ have vertices of degree 5 , so they cannot be subgraphs of $G$. Also, $G$ cannot contain any of the graphs $B_{5}, B_{6}$ since each of them has a pair of vertices of degree four whose transposition is an automorphism of $B_{i}$, and thus of any supergraph of maximum degree 4 . This contradicts the assumption that $m(G)>2$.

It follows that $G$ has to contain at least one of the four graphs $B_{i}, i=1,2,3,4$. We examine each of them as induced subgraphs of the claw-free graph $G$ with $\Delta(G)=4$ and $m(G) \geqslant 4$. It easily turns out for every $i=1,2,3,4$, that if $G$ contains $B_{i}$ as an induced subgraph, then $G$ has to contain a subgraph $I_{i}$, called a basic initial subgraph. All four basic initial subgraphs are presented in Figure 4.

As an example, we describe a method how to obtain $I_{1}$ (see Figure 5 ). Denote the vertex set of $B_{1}$ as $\left\{x_{0}, x_{1}, x_{2}, x_{3}, y_{1}\right\}$. The transposition of vertices $x_{0}, x_{2}$ is an automorphism with motion 2 . Hence, one of these two vertices, say $x_{0}$, has to have another neighbour. It cannot be $y_{1}$, because $B_{1}$ has to be an induced subgraph. Then we join $x_{0}$ with a new vertex $x_{4}$. To avoid a claw $x_{0} ; x_{1}, x_{3}, x_{4}$ (i.e. with $x_{0}$ as the center and $x_{1}, x_{3}, x_{4}$ as the 


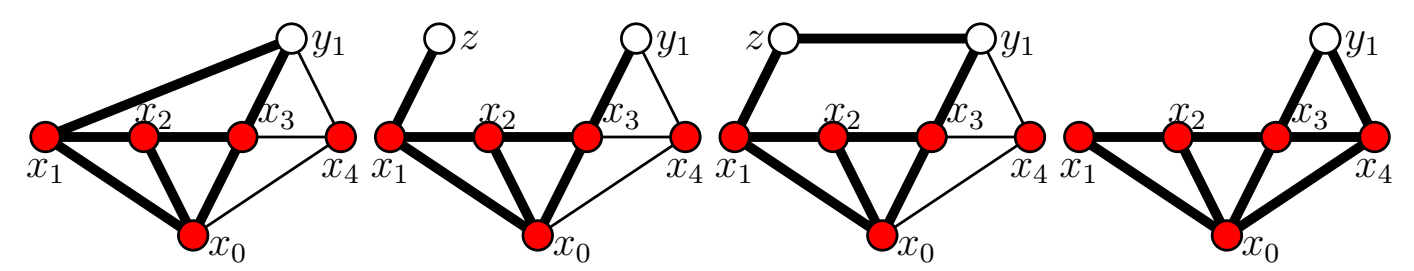

Figure 4: Four basic initial subgraphs $I_{1}, I_{2}, I_{3}, I_{4}$ rooted at $x_{0}$, obtained from $B_{1}, B_{2}$, $B_{3}, B_{4}$, respectively. Subgraphs with bold edges are induced.

leaves), we have to join $x_{4}$ with $x_{1}$ or $x_{3}$. Without loss of generality, we join $x_{4}$ with $x_{3}$. Again, we get a claw $x_{3} ; x_{4}, x_{2}, y_{1}$. If we added the edge $x_{4} x_{2}$, we would obtain two vertices $x_{0}, x_{2}$ of degree 4 with equal closed neighbourhoods. Hence, their transposition would yield the motion of this graph, and thus of any supergraph $G$. It would be 2 , because $\Delta(G)=4$. Therefore, we have to add the edge $x_{4} y_{1}$, thus obtaining the initial basic subgraph $I_{1}$. Similar arguments lead to the other three initial basic subgraphs $I_{2}, I_{3}, I_{4}$.
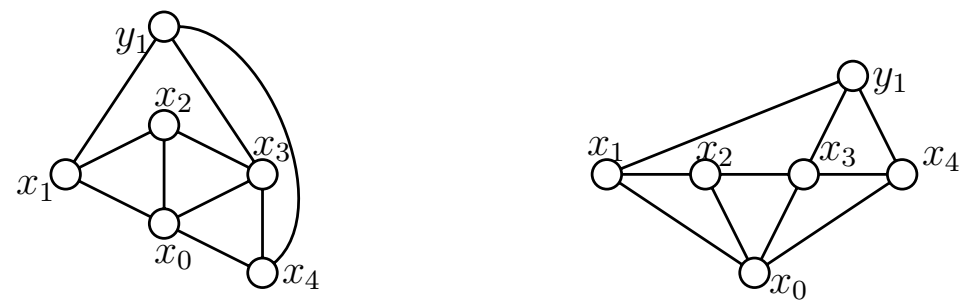

Figure 5: How we get the basic initial subgraph $I_{1}$ from $B_{1}$.

The basic initial graphs $I_{i}, i=1,2,3$, w will be considered as rooted at the vertex $x_{0}$ (see Figure 4). A connected induced subgraph $H$ of the graph $G$ is called an initial graph if it can be obtained from one of the basic initial graphs $I_{i}$ by adding some edges and vertices in such a way that:

- the root $x_{0}$ is of degree 4 in $H$,

- a vertex $v$ belongs to $V(H)$ if and only if $v$ is at distance at most 2 from $x_{0}$ in $G$,

- the induced bold subgraph of $I_{i}$ is still induced in $H$.

Lemma 12. Let $H$ be an initial graph rooted at $x_{0}$. Then the graph induced by the neighborhood of $x_{0}$ is a path $P=x_{1} x_{2} x_{3} x_{4}$.

Proof. Let $H$ be an initial graph obtained from some $I_{i}$. Without loss of generality, it suffices to show that neither $x_{2} x_{4}$ nor $x_{1} x_{4}$ is an edge of $H$.

The edge $x_{2} x_{4}$ does not belong to the graph $H$, because then the vertices $x_{3}$ and $x_{4}$ would be two vertices of degree 4 having the same neighbours in the graph $H-x_{3} x_{4}$. Their transposition would be a graph automorphism, which is contrary to the condition that the graph motion is greater than 2. Observe that this argument remains valid for all basic initial subgraphs $I_{i}$. 
Similarly, in the case $i=1$, the edge $x_{1} x_{4}$ does not belong to the graph, because then the vertices $x_{1}$ and $x_{3}$ would be two vertices of degree 4 having the same neighbours in the graph $H$.

In the case $i=2,3$, if $x_{1} x_{4} \in E(H)$, then either we have the claw $x_{1} ; x_{2}, x_{4}, z$ or $z x_{4} \in E(H)$. But then $d\left(x_{4}\right) \geqslant 5$, a contradiction.

In the case $i=4$, the edge $x_{1} x_{4}$ does not belong to the graph, because both of its ends belong to the bold subgraph.

The idea of the next part of the proof of the Main Theorem is the following. First, we show that every initial graph $H$ can be coloured with two colours, white and red, in such a way that

- $H$ has a red vertex with four red neighbours,

- the only automorphism of $H$ preserving this colouring is the identity.

Then we recursively extend this colouring to an asymmetric colouring $c$ of the whole graph $G$ such that each red vertex outside the initial graph $H$ has at most three red neighbours.

An initial subgraph $H$ rooted at $x_{0}$ is called symmetric if there exists a non-identity automorphism $\alpha \in \operatorname{Aut}(H)$ such that $\alpha\left(x_{0}\right)=x_{0}$. If $H$ is an initial subgraph that is not symmetric, then we colour $x_{0}$ and all four neighbours red, while the vertices of the second sphere at $x_{0}$ white. Hence, $H$ will be fixed by every automorphism of the graph $G$ preserving any colouring of vertices of $G$ satisfying the property that $x_{0}$ is the only red vertex of $G$ with four red neighbours.

Lemma 13. Let $H$ be a symmetric initial graph rooted at $x_{0}$. Then $H$ is isomorphic to one of the following twelve graphs $\mathrm{H}_{1}, \mathrm{H}_{2}, \mathrm{H}_{2}^{+}, \mathrm{H}_{3}, \mathrm{H}_{4}, \mathrm{H}_{5}, \mathrm{H}_{5}^{+}, \mathrm{H}_{6}, \mathrm{H}_{7}, \mathrm{H}_{8}, \mathrm{H}_{9}, \mathrm{H}_{9}^{+}$ presented in Figure 6.

Proof. Recall that $x_{0}$ is of degree 4 and its neighbours (i.e. its first sphere) induce a path $P=x_{1} x_{2} x_{3} x_{4}$. The vertices at distance 2 from $x_{0}$ (i.e. its second sphere) will be denoted by $y_{j}$. Let $\alpha$ be a non-identity automorphism of $H$ such that $\alpha\left(x_{0}\right)=x_{0}$. Since the image of the first sphere of a fixed vertex is again its first sphere, we have two possibilities for the realization of the fact that $\alpha(P)=P$. We shall consider these two cases separately.

Case A. The automorphism $\alpha$ is not the identity on the path $P$, that is, $\alpha_{\mid P}=$ $\left(x_{1} x_{4}\right)\left(x_{2} x_{3}\right)$.

This condition implies that the second sphere contains another vertex, say $y_{2}$, such that $\alpha\left(y_{1}\right)=y_{2}$ (see Figure 4 and Figure 6 ). In consequence, the edges $y_{2} x_{1}, y_{2} x_{2}$ belong to $H$. We will consider some cases depending on the number of vertices belonging to the second sphere.

Subcase (1). There are only two vertices in the second sphere.

Let us note that in this case, $H$ is a supergraph of the graph $I_{1}$ or of the graph $I_{4}$. If $H$ is a supergraph of $I_{1}$, then in order to avoid the claw with vertices $x_{1} ; x_{0}, y_{1}, y_{2}$, we have $y_{1} y_{2} \in E(H)$. Since $\alpha\left(x_{1}\right)=x_{4}$ and $\alpha\left(y_{1}\right)=y_{2}$, the edge $x_{4} y_{2}$ also belongs to $E(H)$, and $H$ is isomorphic to $H_{1}$ (see Figure 6). 

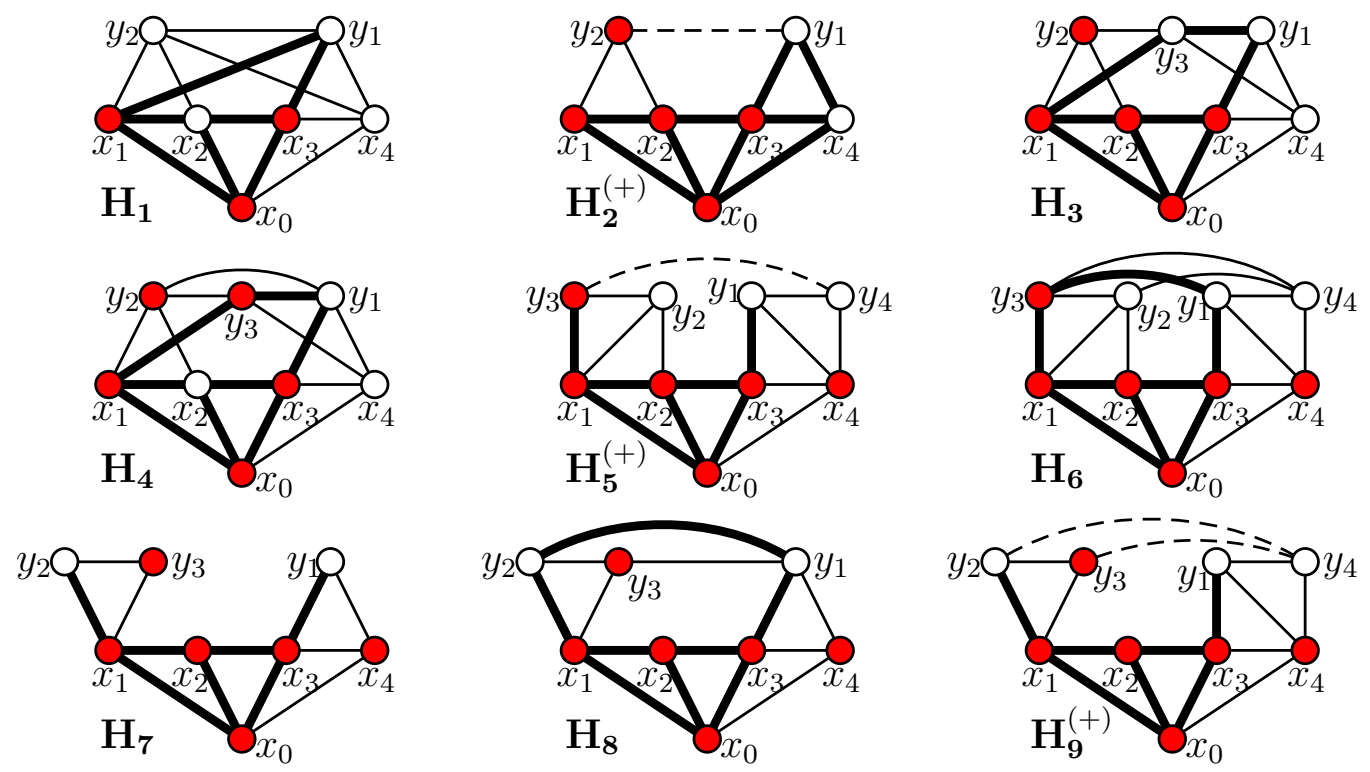

Figure 6: Initial symmetric subgraphs with adequate vertex colourings. Bold subgraphs are induced. A graph having dashed edges stands actually for two graphs, one without and the second one with dashed edges.

If $H$ is a supergraph of $I_{4}$ and there is no additional edge, then we get $H_{2}$. The additional edge $y_{1} y_{2}$ changes it into $H_{2}^{+}=H+y_{1} y_{2}$.

Subcase (2). There are exactly three vertices in the second sphere.

Let us denote this third vertex by $y_{3}$. It is easy to see that this vertex should be fixed by $\alpha$. Since the vertices $x_{2}$ and $x_{3}$ are already of degree 4 , then $y_{3}$ has to be attached by an edge to $x_{1}$ and $x_{4}$. Next, in order to avoid the claw $x_{1} ; y_{3}, y_{2}, x_{0}$, we have $y_{3} y_{2} \in E(H)$. By symmetry, also $y_{3} y_{1} \in E(H)$, and we get the graph $H_{3}$ (see Figure 6 ). If additionally, the edge $y_{1} y_{2}$ belongs to $H$, we get $H_{4}$. Observe that $H_{3}$ and $H_{4}$ are supergraphs of the graph $I_{3}$ or $I_{4}$.

Subcase (3). There are four vertices in the second sphere.

Denote by $y_{3}$ and $y_{4}$ two new vertices belonging to the second sphere and adjacent to $x_{1}$ and $x_{4}$, respectively. Then also $y_{3} y_{2} \in E(H)$. For, we would have the claw induced by $x_{1} ; y_{3}, y_{2}, x_{0}$. By symmetry, also $y_{1} y_{4} \in E(H)$. This graph is denoted by $H_{5}$. It can be considered as a supergraph of $I_{2}$ or $I_{4}$, and the same applies to the graph with an additional edge between $y_{3} y_{4}$, i.e. the graphs $H_{5}^{+}=H_{5}+y_{3} y_{4}$.

However, if we would like to add another edge to $H_{5}$, e.g. $y_{3} y_{1}$, we would have to consider a graph $H$ as a supergraph of the graph $I_{3}$ or $I_{4}$. By symmetry, our graph would also have the edge $y_{2} y_{4}$, and also $y_{3} y_{4}$ to avoid the claw $y_{2} ; y_{4}, y_{3}, x_{2}$. This way we arrive at the graph $H_{6}$.

Observe that the edge $y_{2} y_{1}$ cannot be added do $H_{5}$. For, either we would have the claw $y_{2} ; y_{1}, y_{3}, x_{2}$, or the edge $y_{3} y_{1}$ would make the degree of the vertex $y_{1}$ greater than 4 .

Case B. The automorphism $\alpha$ fixes the path $P$.

Because $x_{3}$ is fixed, the vertex $y_{1}$, belonging to the second sphere, is also fixed by $\alpha$, 
for, there is only one edge going out of $x_{3}$ to the second sphere. This implies that if $x_{4}$ is adjacent to a vertex on the second sphere, that this vertex is also fixed by $\alpha$.

Because $\alpha \in \operatorname{Aut}(H) \backslash\{\operatorname{id}\}$, the graph $H$ has to contain two vertices of the second sphere, say $y_{2}, y_{3}$, with $\alpha\left(y_{2}\right)=y_{3}$ and $\alpha\left(y_{3}\right)=y_{2}$. None of them is adjacent to $x_{2}$, since then there would be only one edge joining $x_{2}$ to the second sphere and the neighbour of $x_{2}$ in the second sphere would be fixed.

So, both vertices $y_{2}, y_{3}$ are adjacent to $x_{1}$, and, because of the possible claw $x_{1} ; y_{2}, y_{3}$, $x_{0}$, the graph $H$ should contain the edge $y_{2} y_{3}$.

If $H$ does not contain other edges anymore, we get a graph isomorphic to $H_{7}$ with $I_{2}$ (or $I_{4}$ ) as an initial subgraph.

If $H$ contains the edge $y_{1} y_{3}$, then it contains also the edge $y_{1} y_{2}=\alpha\left(y_{1} y_{3}\right)$ and we get a graph isomorphic to $H_{8}$. This time, however, our graph is not a supergraph of $I_{2}$ but of $I_{3}$ or $I_{4}$.

Finally, if $H$ has another vertex adjacent to $x_{4}$, say $y_{4}$, then it also has the edge $y_{1} y_{4}$. If we do not have any other edges then we get $H_{9}$ (see Figure 6). It is possible, however, that $H$ also has edges connecting $y_{4}$ with $y_{2}$ and $y_{3}$. Then our graph takes the form of $H_{9}^{+}=H_{9}+y_{2} y_{4}+y_{3} y_{4}$. Both $H_{9}$ and $H_{9}^{+}$can be treated as graphs created from $I_{2}$ or $I_{4}$.

Let $H$ be an initial subgraph. If $H$ is symmetric, then $H$ is one of the twelve graphs specified in Lemma 13. Note that the graphs $H_{1}$ and $H_{4}$ are 4-regular, so none of them can be a proper subgraph of a graph $G$ with $\Delta(G) \leqslant 4 . H_{1}$ is the complement of the cycle $C_{7}=x_{0} y_{1} x_{2} x_{4} x_{1} x_{3} y_{2} x_{0}$ and it is easy to see that the colouring shown in Figure 6 stabilizes this cycle (and thus its complement).

As for $H_{4}$, observe that $x_{2}$ is the only white vertex with four red neighbours inducing a path $P_{4}$. This vertex and its neighbours are fixed because the path $P_{4}$ cannot be reversed. Indeed, the end-vertices of $P_{4}$ have different numbers of white neighbours. Then it is easily seen that also the remaining vertices of the second sphere at $x_{2}$ cannot be permuted.

This reasoning can be repeated in the case of graphs $H_{2}, H_{2}^{+}, H_{3}$. There $x_{2}$ is the only red vertex with four red neighbours inducing a path $x_{3} x_{0} x_{1} y_{2}$. Hence, $x_{2}$ is fixed. Furthermore, the path $x_{3} x_{0} x_{1} y_{2}$ cannot be reversed, since $x_{0}$ cannot be mapped into $x_{1}$. Indeed, $x_{0}$ has a white neighbour in the graphs $H_{2}$ and $H_{2}^{+}$while $x_{1}$ does not. In the graph $H_{3}$, the vertex $x_{3}$ cannot be mapped into $y_{2}$ because $x_{3}$ has only one red neighbour while $y_{2}$ has at least two red neighbours in the graph $G$.

Observe that for each of the remaining seven initial subgraphs, the colouring presented in Figure 6 is also an asymmetric colouring of $H$. There, $x_{0}$ is the only red vertex with four red neighbours inducing a path. This red vertex and its neighbours are fixed because the path $P_{4}$ cannot be reversed. Indeed, in each case, the end-vertices of $P_{4}$ have different pairs of numbers of white and red neighbours. Then it is easily seen that also the remaining vertices of the second sphere cannot be permuted.

We recursively extend the colouring of an initial subgraph $H$ to obtain an asymmetric colouring $c$ of vertices of the graph $G$ with a unique red vertex with four red neighbours, the one contained in the initial subgraph $H$. In our procedure, every coloured vertex becomes fixed by $c$ at once. Moreover, every vertex coloured red gets a white neighbour. 
Suppose we have already coloured vertices of a certain connected induced subgraph $H$ of the graph $G$ such that the only automorphism of $H$ preserving this colouring is the identity. Consequently, each vertex of $H$ will be fixed by every automorphism of $H$ preserving any extension $c$ of this partial colouring, provided there will be no other red vertex with four red neighbours.

Let $\partial H$ denote the set of vertices of $H$ having a neighbour outside $H$. In our procedure, each vertex of $\partial H$ has also a neighbour in $H-\partial H$. In particular, this is the case for any initial subgraph $H$. Hence, each pair of vertices outside $H$ with a common neighbour in $\partial H$ have to be adjacent to avoid a claw.

We now colour all vertices of distance one from $H$, and in some special cases, some vertices of distance 2 from $H$.

First, we consider the vertices of $H$ that have exactly one neighbour outside $H$. These neighbours are already fixed by $c$, and we colour them white. Next, we consider all vertices $v \in V(H)$ that have exactly two neighbours $u_{1}, u_{2} \notin V(H)$. It follows that $u_{1} u_{2} \in E(G)$, because $G$ is claw-free, while $v$ has a neighbour in $H-\partial H$. If $u_{1}$ or $u_{2}$ is already coloured, then both of these two vertices are fixed. We colour the possibly uncoloured vertex white. Otherwise, we colour $u_{1}, u_{2}$ with two different colours. Note that every vertex we colour is either white or has a white neighbour.

Finally, let a vertex $v \in V(H)$ have three neighbours $u_{1}, u_{2}, u_{3}$ outside $H$. They induce a triangle $K_{3}$, because $G$ is claw-free. At least two of them are of degree 4, and no two of them have a common neighbour outside the clique $K_{4}=G\left[\left\{v, u_{1}, u_{2}, u_{3}\right\}\right]$, because $m(G)>2$. If three of the vertices $u_{1}, u_{2}, u_{3}$ are already coloured, then all three are fixed by $c$ and we have nothing to do. If two of the vertices $u_{1}, u_{2}, u_{3}$ are already coloured, then again all three of them are fixed. The only uncoloured vertex gets colour white. If only one of them is already coloured, then it is fixed and we colour the two other vertices white and red. Suppose now that all three vertices $u_{1}, u_{2}, u_{3}$ are still uncoloured. Observe that they do not have neighbours in $H$ other than $v$. We colour two of them that have degree 4 with two distinct colours, and the third one, say $u_{3}$, we colour white. If $u_{3}$ is also of degree 4 , then we colour the neighbours of white vertices (possibly they are of distance two from $H$ ) with two distinct colours. Thus all three vertices $u_{1}, u_{2}, u_{3}$ are fixed.

Observe that in our procedure of extending the colouring, every new red vertex obviously gets a white neighbour. Moreover, if $H$ is an initial subgraph that is not symmetric, then every vertex having a neighbour outside $H$ is white because the second sphere at $x_{0}$ is white. If $H$ is a symmetric initial graph, then the only red vertex that may have neighbours outside $H$ and does not have a white neighbour yet, is the vertex $y_{2}$ of degree 2 in $\mathrm{H}_{2}$. In our procedure, $y_{2}$ cannot have two new red neighbours.

We thus obtained an asymmetric colouring of a new larger subgraph of $G$ that in the next stage plays the role of $H$. Finally, after a countable number of stages, we obtain a distinguishing colouring of $G$ with two colours. 


\section{References}

[1] M. Albertson and K. Collins, Symmetry breaking in graphs. Electron. J. Combin., 3:\#R18, 1996.

[2] L. Babai, Problem presented at the workshop on Symmetry Breaking in Discrete Structures. Casa Matemática Oaxaca (CMO), Mexico, September 2018.

[3] L. Beineke, Derived Graphs and Digraphs. Beiträge zur Graphentheorie, Teubner, Leipzig, 17-33, 1968.

[4] I. Broere and M. Pilśniak, The distinguishing index of the Cartesian product of countable graphs. Ars Math. Contemp., 13:15-21, 2017.

[5] P. Cameron, Regular orbits of permutation groups on the power set. Discrete Math., 62:307-309, 1986.

[6] K. Collins and A. Trenk, Nordhaus-Gaddum Theorem for the Distinguishing Chromatic Number. Electron. J. Combin., 20(3):\#P46, 2013.

[7] S. Hüning, W. Imrich, J. Kloas, H. Schreiber and T. W. Tucker, Distinguishing graphs of maximum valence 3. Electron. J. Combin. 26(4):\#P4.36, 2019.

[8] W. Imrich, S. Klavžar and V. Trofimov, Distinguishing infinite graphs. Electron. J. Combin., 14: \#R36, 2007.

[9] W. Imrich and T. Tucker, Asymmetrizing trees of maximum valence $2^{\aleph_{0}}$. Monatsh. Math., 192:615-624, 2020.

[10] H.Jung, Zu einem Isomorphiesatz von H. Whitney für Graphen. Math. Ann., 164:270-271, 1966.

[11] R. Kalinowski and M.Pilśniak, Distinguishing graphs by edge-colourings. European J. Combin., 45:124-131, 2015.

[12] S. Klavžar, T.-L. Wong and X. Zhu, Distinguishing labelings of group action on vector spaces and graphs. J. Algebra, 303:626-641, 2006.

[13] F. Lehner, Breaking graph symmetries by edge colourings. J. Combin. Theory, Ser. B, 127:205-214, 2017.

[14] F. Lehner, M. Pilśniak and M. Stawiski, Distinguishing infinite graphs with bounded degrees. preprint, 2019.

[15] F. Lehner and S.Smith, On symmetries of edge and vertex colourings of graphs. Discrete Math., 343(9):111959, 2020.

[16] F. Lehner and G. Verret, Distinguishing numbers of finite 4-valent vertex-transitive graphs. Ars Math. Contemp., 19(2):172-187, 2020.

[17] M. Pilśniak, Improving upper bounds for the distinguishing index. Ars Math. Contemp., 13:259-274, 2017.

[18] M. Pilśniak and M. Stawiski, The Optimal General Upper Bound for the Distinguishing Index of Infinite Graphs. J. Graph Theory, 93:463-469, 2020.

[19] A. Russell and R. Sundaram, A note on the asymptotics and computational complexity of graph distinguishability. Electron. J. Combin., 5:\#R23, 1998.

[20] T. Tucker. Distinguishing maps. Electron. J. Combin., 18(1):\#P50, 2011. 